\title{
Partial resistance to clubroot in Arabidopsis is based on changes in the host primary metabolism and targeted cell division and expansion capacity
}

\author{
Mélanie Jubault • Christine Lariagon • \\ Ludivine Taconnat • Jean-Pierre Renou • \\ Antoine Gravot • Régine Delourme • \\ Maria J. Manzanares-Dauleux
}

Received: 18 July 2012 / Revised: 21 January 2013 /Accepted: 4 February 2013 /Published online: 19 February 2013

(C) The Author(s) 2013. This article is published with open access at Springerlink.com

\begin{abstract}
To date, studies of the molecular basis of disease resistance mainly focused on qualitative resistance. However, deciphering mechanisms underlying quantitative resistance could lead to insights into the relationship between qualitative and quantitative resistance and guide the utilization of these two types of resistance to produce durably resistant cultivars. A functional genomics approach, using the CATMA wholegenome microarray, was used to detect changes in gene expression associated with partial quantitative resistance in the
\end{abstract}

Electronic supplementary material The online version of this article (doi:10.1007/s10142-013-0312-9) contains supplementary material, which is available to authorized users.

M. Jubault • M. J. Manzanares-Dauleux

Agrocampus Ouest, UMR1349 IGEPP, 35000 Rennes, France

M. Jubault $\cdot$ A. Gravot $\cdot$ M. J. Manzanares-Dauleux

Université Européenne de Bretagne, Rennes, France

C. Lariagon $\cdot$ R. Delourme

INRA, UMR1349 IGEPP, 35653 Le Rheu, France

L. Taconnat $\cdot$ J.-P. Renou

UMR INRA 1165-CNRS 8114-UEVE, Unité de Recherche en Génomique Végétale, Université d'Evry-Val-d'Essone, CP 5708, 91057 Evry Cedex, France

\section{A. Gravot}

Université Rennes 1, UMR1349 IGEPP, 35000 Rennes, France

M. J. Manzanares-Dauleux ( $\square)$

UMR 1349 IGEPP INRA, Agrocampus Ouest Rennes, Université Rennes 1, BP35327, 35653 Le Rheu Cedex, France e-mail: maria.manzanares@agrocampus-ouest.fr

Present Address:

J.-P. Renou

UMR IRHS, 42 rue Georges Morel,

49071 Beaucouzé Cedex, France
Arabidopsis thaliana-Plasmodiophora brassicae pathosystem. The time course of transcript abundance during partial clubroot resistance response was monitored at the whole plant level, and direct comparisons between partial resistance and susceptibility responses were made using the same host genotype. An increasingly complex host response was revealed, as was the differential influence of $P$. brassicae infection on the transcription of Arabidopsis genes according to the isolate used. We observed, at the transcriptomic level, that metabolic diversion by the pathogen was reduced or delayed, classical plant defense responses were induced earlier and/or more strongly, and cell enlargement and proliferation were actively inhibited in the partial quantitative resistance response compared to the susceptible one.

Keywords Clubroot - Quantitative resistance - Arabidopsis . Plasmodiophora brassicae · Microarray

\section{Introduction}

Clubroot, caused by the obligate biotrophic protist Plasmodiophora brassicae Woron., is one of the most important diseases of Brassica crops, causing annual losses of 10 $15 \%$ worldwide (Dixon 2009). The life cycle of this soilborne pathogen can be divided into two phases: a primary phase in which events are confined to the root hairs and a secondary phase that occurs in the cortex and the stele of the hypocotyl and roots of the infected plants. During the second phase, multinucleate plasmodia cause the hypertrophy (abnormal cell enlargement) and hyperplasia (uncontrolled cell division) of infected roots into characteristic clubs (Ingram and Tommerup 1972). These obstruct nutrient and water transport, stunt the growth of the plant, and consequently reduce crop 
yield and quality. Since the pathogen survives in the soil as resting spores readily transmittable and potentially viable for up to 15 years (Wallenhammar 1996), successful management of clubroot requires integrated control. The combination of adapted cropping practices as well as chemical and biological control methods is now a feasible strategy for the management of clubroot in Brassica (reviewed in Donald and Porter 2009); however, plant resistance is still the most powerful tool for combating clubroot disease (Diederichsen et al. 2009). Both qualitative and quantitative clubroot resistances were identified in different Brassicaceae species, including the three most economically important Brassica species: Brassica napus, Brassica rapa, and Brassica oleracea (reviewed in Piao et al. 2009). However, the commercial resistant cultivars from these species received primarily a single, dominant, and racespecific resistance gene, and several examples have now demonstrated the rapid adaptation of $P$. brassicae to widespread mono- or oligogenic clubroot resistance sources (Diederichsen et al. 2009). Although defined as a compatible host-pathogen interaction, partial resistance does limit the extent of the disease, either by rate-limiting pathogen multiplication or by reducing symptom severity. This form of resistance, frequently under polygenic control, is important for crop improvement and can be selected, often constituting an additional layer of resistance in the absence of R-mediated resistance and leading to high levels of phenotypic resistance (Poland et al. 2009). Furthermore, because it is controlled by multiple genes with small effects (leading to lower selection pressure on the pathogen) and/or is presumed to have a broader specificity, quantitative resistance should be overcome more slowly by the pathogen and appears to be an alternative for the development of durable host plant resistance (Boyd 2006; Brun et al. 2010; Palloix et al. 2009).

Up until now, the identification of components required for quantitative partial clubroot resistance was mainly based on quantitative trait loci mapping, both in cultivated species (Piao et al. 2009; Manzanares-Dauleux et al. 2000a, 2003; Rocherieux et al. 2004) and the model plant Arabidopsis thaliana (Jubault et al. 2008b). Functional studies on clubroot have mainly been conducted on the physiopathological mechanisms involved in the infection of susceptible hosts by P. brassicae (reviewed in Ludwig-Muller et al. 2009), but only a few studies, done on Arabidopsis, have focused on the mechanisms controlling quantitative resistance. Jubault et al. (2008a) reported strikingly different arginine catabolism signatures between susceptible and partially resistant plants. In particular, susceptible plants were characterized by a massive induction of arginase during the later stages of disease. This huge arginase induction actually constitutes a basal defense mechanism by reducing hormone-triggered cellular proliferation (Gravot et al. 2012). The lower induction of arginase in the partially resistant plants during $P$. brassicae infection reflects the attenuation or the delay of the pathogen influence on host metabolism in partially resistant plants compared to the situation in susceptible plants (Jubault et al. 2008a) and is more likely the result of partial clubroot resistance than its cause. Moreover, Gravot et al. (2011) showed that, although partial resistance to clubroot is not directly based on trehalose catabolism capacity, it is to some extent related to the tolerance to trehalose accumulation in the partially resistant accession Bur- 0 . However, the mechanisms underlying clubroot partial resistance currently remain largely unknown.

To gain further insight into this resistance type, a complementary approach is to identify candidate genes whose expression changes are in association with partial resistance, suggesting functional involvement. Using microarray technology, genome-wide information about patterns of gene expression during interactions between Arabidopsis or cultivated species and a variety of different pathogens was previously obtained. Analysis of host gene expression using microarrays provided significant insight into the transcriptional responses triggered during either R-mediated resistance (complete resistance) or basal defense (susceptibility; Tao et al. 2003; Marathe et al. 2004; Siemens et al. 2006; Jammes et al. 2005; Huibers et al. 2009; Radwan et al. 2011; AbuQamar et al. 2006; Swarbrick et al. 2008; Thilmony et al. 2006; Ditt et al. 2006; Agarwal et al. 2011; Mazarei et al. 2011). Microarray analyses were previously carried out to study $A$. thaliana $-P$. brassicae interactions; however, these were only done on disease development in the susceptible accession Columbia (Siemens et al. 2006; Agarwal et al. 2011).

Here, we report a large-scale gene expression profiling study of partial clubroot resistance in $A$. thaliana using the complete Arabidopsis transcriptome microarray (CATMA) chips (Crowe et al. 2003; Hilson et al. 2004). The Bur-0 accession is partially resistant to the isolate eH; however, it is fully susceptible to the isolate $\mathrm{e}_{2}$. This finding can thus be exploited to investigate, using the same host genotype, the transcriptional changes associated with these two levels of compatible interaction and determine specific molecular patterns associated with a partial resistance response compared to a susceptible one. At the transcriptomic level, the partial resistance response is associated with (1) a reduced or delayed host metabolic diversion by the pathogen, (2) an earlier and/or stronger induction of usual plant defense responses, and (3) a reduced expression of genes involved in cell enlargement and proliferation.

\section{Materials and methods}

\section{Pathogen}

The selection isolates eH and $\mathrm{e}_{2}$ (Fähling et al. 2003) belong to the $P$. brassicae pathotype $\mathrm{P} 1$, according to the host differential set established by Somé et al. (1996). They were 
kindly provided by J. Siemens (University of Dresden, Germany).

\section{Plant materials}

Seeds from Arabidopsis accession Bur-0 (172AV) were obtained from the Versailles Resource Centre. This accession is partially resistant to the eH isolate (Alix et al. 2007) and susceptible to the $\mathrm{e}_{2}$ isolate, respectively. B. napus ssp. oleifera cv. "Nevin" (ECD6), B. napus ssp. rapifera cv. "Wilhelmsburger" (ECD10), and B. napus ssp. oleifera (Brutor), which constitute the host differential set established by Somé et al. (1996), and the highly clubroot susceptible B. rapa ssp. pekiniensis cv. "Granaat" (ECD5) were included as controls in each clubroot test.

\section{Experimental design and clubroot tests}

Two independent studies were performed (Fig. 1). In the first study, comparisons were made between control plants and plants inoculated with the eH isolate (comparisons 1-3). Inoculated and control plants were harvested at three time points: 1, 2, and 7 days post-inoculation (dpi; respectively stages 1.04 and 1.08; Boyes et al. 2001). The second study was performed only at $7 \mathrm{dpi}$, and comparisons were made between plants inoculated with either the $\mathrm{eH}$ or the $\mathrm{e}_{2}$ isolate (comparison 6). Each experiment was repeated twice.

Arabidopsis seeds were placed on wet blotting paper in Petri dishes at $4{ }^{\circ} \mathrm{C}$ for 3 days to synchronize germination; then, seeds were individually sown in 4-cm diameter pots containing a two thirds compost/one third vermiculite mix sterilized by autoclaving. Arabidopsis plants were grown under controlled environmental conditions (16-h light at $22{ }^{\circ} \mathrm{C}$ and 8 -h dark at $19{ }^{\circ} \mathrm{C}$ ) and inoculated 7 days after germination (stage 1.04; Boyes et al. 2001). The inoculum was prepared according to Manzanares-Dauleux et al. (2000a), and inoculation was performed by applying $1 \mathrm{~mL}$ of resting spore suspension ( $10^{7}$ spores per milliliter) to the crown of each seedling. The resting spore suspension was replaced by distilled water for the control plants. Thirty individual plants were collected per analysis point. Plants were thoroughly rinsed in different baths of water, frozen in liquid nitrogen, and stored at $-80{ }^{\circ} \mathrm{C}$ until RNA isolation. To check that the inoculation was successful, clubroot susceptibility was evaluated in each test from 21 dpi (from stage 3.90 to 6.50 ; Boyes et al. 2001) and symptoms were recorded using the scale previously described for B. oleracea (Manzanares-Dauleux et al. 2000b): 0-no visible swelling; 1 -very slight swelling usually confined to lateral roots; 2 - moderate swelling on lateral roots and taproot; $2+$ severe clubs on all roots, but some roots remain; 3-no root left, only one big gall. A disease index (DI) was calculated as described by Manzanares-Dauleux et al. (2000b): $\mathrm{DI}=$ $\left(\mathrm{n}_{1} \times 25+\mathrm{n}_{2} \times 50+\mathrm{n}_{2^{+}} \times 75+\mathrm{n}_{3} \times 100\right) / \mathrm{N}$, where $n_{i}$ is the number of plants in the symptom class $i$ and $N$ the total number of plants tested. A line with a DI of zero is completely resistant and develops no clubroot symptoms, while a line with a DI of 100 is highly susceptible. Susceptibility to clubroot was also quantified by evaluation of the $\mathrm{Ga} / \mathrm{La}$ pathological index [the ratio between gall area ( $\mathrm{Ga}$, in square centimeters) and rosette leaf area (roughly evaluated by the square of the longest leaf length - La, in square centimeters)] using image analysis, as previously described in Gravot et al. (2011).

\section{RNA isolation}

For each analysis point, total RNA was extracted from approximately $30 \mathrm{mg}$ of 30 pooled plants using the SV
Fig. 1 Typical symptoms during partial resistance and susceptibility responses to P. brassicae in Arabidopsis. a Tiny clubs are mainly confined to the secondary root system in the eH-inoculated plants (partial resistance), whereas the main and secondary root systems are replaced by a big club in the $e_{2}$-inoculated plants (susceptible response) (b)
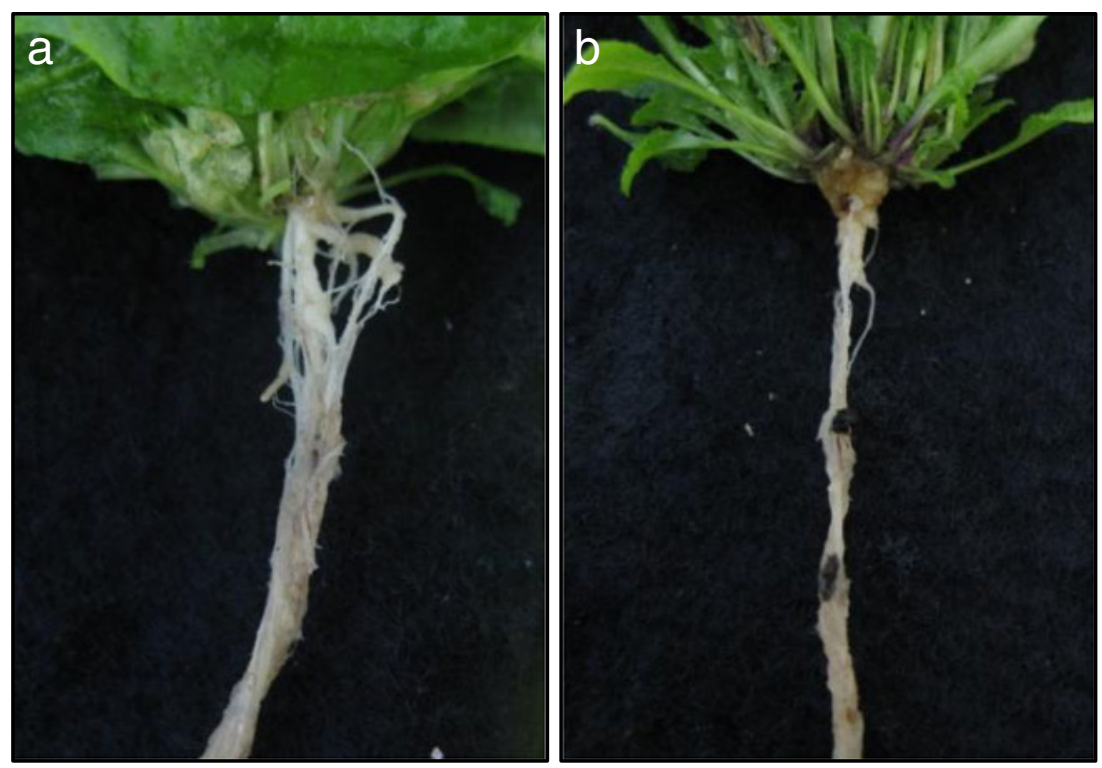
Total RNA Isolation kit (Promega, Madison, WI). Any remaining genomic DNA was removed by digestion with DNase I (DNA-free ${ }^{\mathrm{TM}}$, Ambion ${ }^{\circledR}$, Austin, TX). RNA integrity was checked with the Bioanalyzer from Agilent (Waldbroon, Germany).

\section{Microarray analyses}

Microarray analyses were performed with the A. thaliana CATMA array containing 24,576 nuclear gene-specific tags (GST) corresponding to 22,089 nuclear genes, including 21,612 AGI-predicted genes and 477 Eugene-predicted genes (Allemeersch et al. 2005; Hilson et al. 2004). The GST (which are between 150 and $500 \mathrm{bp}$ in length and show no more than $70 \%$ identity with any other sequence in the genome) were spotted on UltraGAPS slides (Corning, NY) using a BioRobotics Microgrid II TAS spotter (Genomic Solution, Huntingdon, UK). Detailed information about CATMA and database access can be found online (Crowe et al. 2003).

Six comparisons were performed during the time course analysis as described in Fig. 2. The array was hybridized simultaneously with cRNA from both samples labeled with Cy3 and Cy5 fluorescent dyes, respectively. For each comparison, a repeat was carried out using a second set of samples and a dye swap experiment to avoid dye bias and gene-specific dye bias (Martin-Magniette et al. 2005). Therefore, a total of 24 arrays were hybridized.

The complete microarray procedure has been fully described before (de Jong et al. 2006). Briefly, for each condition, mRNA from isolated RNA was amplified with the MessageAmp aRNA kit (Ambion, Austin, TX). Then, amplified mRNA was used as a template to synthesize modified cDNA with SuperScript II (Invitrogen, Carlsbad, CA) and random nonamers (Gene Link, Westchester County, NY) with the incorporation of cy3-dUTP and cy5-dUTP (NEN, Boston, MA; Puskas et al. 2002). Samples were combined, purified, and concentrated with YM30 Microcon columns (Millipore, Bedford, MA). The probes were hybridized overnight on CATMA arrays which were then scanned using a GenePix 4000A scanner (Axon Instruments, Foster City, CA). Spot intensities of the scans were determined by GenePix Pro 3.0 software (Axon Instruments).

Fig. 2 Experimental design for complete Arabidopsis transcriptome microarray (CATMA) transcript profiling of partial clubroot resistance
Statistical analysis of microarray data

Analysis of spot intensities from the CATMA arrays and applied statistics were performed as described previously (de Jong et al. 2006). Differentially expressed genes were selected based on a Bonferroni $p$ value $<5 \%$.

Microarray data

Microarray data from this article were deposited at ArrayExpress (http://www.ebi.ac.uk/arrayexpress/; accession EMEXP-363) and CATdb (http://urgv.evry.inra.fr/CATdb/; Project RA03-05_Clubroot**) according to the "Minimum Information About a Microarray Experiment" standards. Functional categories of differentially expressed genes were based on the Functional Catalogue (FunCat) scheme from the MatDB database (MIPS A. thaliana Database; http:// mips.gsf.de/proj/funcatDB). Major metabolic pathways were analyzed using the MAPMAN software (Thimm et al. 2004).

\section{Real-time RT-PCR}

First-strand cDNA was synthesized with Superscript ${ }^{\mathrm{TM}}$ II Reverse Transcriptase (Invitrogen) and oligo(dT) ${ }_{15}$ (Promega Corp.). For each gene, primers for real-time RTPCR were designed on GSTs (Hilson et al. 2004) with Primer Express ${ }^{\circledR}$ v1.5 software (Applied Biosystems) and synthesized by Eurogentec. The genes, as well as the sequence of their specific oligonucleotides, are presented in Electronic supplementary material (ESM) Table 1. Duplicate quantitative assays were performed on $3 \mu \mathrm{L}$ of 1/40 diluted cDNA using the SYBR-Green PCR Master kit (Applied Biosystems) with the ABI PRISM ${ }^{\circledR} 7700$ Sequence Detection system (Applied Biosystems). To check the annealing specificity of each oligonucleotide, melting curve analysis $\left(55-94{ }^{\circ} \mathrm{C}\right)$ was carried out at the end of amplification. For calculations, a standard curve was determined for each gene using different dilutions of the cDNA products. The expression levels for each target gene were then quantified following normalization to Actin8, the endogenous reference.

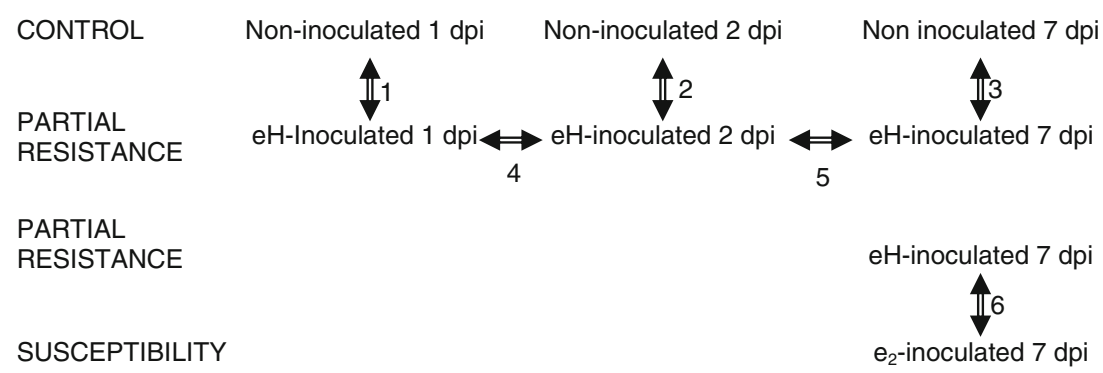


Table 1 Evaluation of Bur-0 gall and leaf areas during partial resistance (inoculated with the eH isolate) and susceptibility (inoculated with the $\mathrm{e}_{2}$ isolate) responses to $P$. brassicae

\begin{tabular}{llll}
\hline Isolate & $\begin{array}{l}\text { Gall } \\
\text { area, Ga }\end{array}$ & $\begin{array}{l}\text { Rosette } \\
\text { leaf area, } \mathrm{La}\end{array}$ & $\begin{array}{l}\mathrm{Ga} / \mathrm{La} \text { pathological } \\
\text { index }\end{array}$ \\
\hline $\mathrm{eH}$ & $0.06 \pm 0.03$ & $25.6 \pm 1.76$ & $13.63 \pm 8.41$ \\
$\mathrm{e}_{2}$ & $0.13 \pm 0.01$ & $16.8 \pm 1.02$ & $49.09 \pm 3.2$ \\
\hline
\end{tabular}

Means are estimated from 18 plants at $28 \mathrm{dpi}$. The $\mathrm{Ga} / \mathrm{La}$ pathological index reflects the ratio between $\mathrm{Ga}$ and rosette La. Results are reported in square centimeters \pm standard deviation

\section{Results}

Bur-0 is partially resistant to the $\mathrm{eH}$ isolate, but susceptible to the $\mathrm{e}_{2}$ isolate

The behavior of the Bur-0 accession was estimated from 21 dpi with $P$. brassicae isolates. As previously reported (Jubault et al. 2008b; Alix et al. 2007), the Bur-0 accession had an intermediate behavior in response to inoculation with the eH isolate, with a mean DI of 66 at 21 dpi. Bur-0 plants infected with this isolate typically showed only tiny clubs confined mainly to the secondary root systems and welldeveloped green rosettes (Fig. 1a and Table 1). On the contrary, Bur-0 was susceptible in response to inoculation with the $e_{2}$ isolate, with a mean DI of 90 at 21 dpi. The plants infected with this isolate exhibited a big club replacing the main and secondary root systems and smaller rosettes (Fig. 1b and Table 1). A set of differential hosts, including susceptible and resistant genotypes of different Brassica species, was also evaluated at 49 dpi to characterize the isolate's pathogenicity. This confirmed that both isolates, eH and $\mathrm{e}_{2}$ (Fähling et al. 2003), used in this study belong to the P. brassicae pathotype P1 (Somé et al. 1996).

Analysis of global changes in gene expression in partial resistance and susceptibility responses to $P$. brassicae infection

We know relatively little about changes in gene expression that occur during partial resistance response to $P$. brassicae infection and their specificity in comparison to the susceptible response. Thus, to gain insights into the transcriptional changes specifically associated with partial resistance, genome-wide expression analyses were carried out at the whole-plant level on the Bur-0 accession infected either with water, eH, or $\mathrm{e}_{2}$ isolates. First, to investigate the timing and extent of transcriptional changes associated with partial clubroot resistance response, comparisons were made between the transcript profiles of Bur- 0 plants inoculated with the isolate $\mathrm{eH}$ and water-inoculated plants (i.e., control plants) over the time course of infection. To specifically relate host responses to the pathogen life cycle, comparisons were made during the symptomless phase at 1, 2 (corresponding to the primary phase), and $7 \mathrm{dpi}$ (corresponding to the initiation of secondary infection in the cortex; Mithen and Magrath 1992; Puzio et al. 2000; Devos et al. 2006; Fig. 2, comparisons 1-3). Statistical analysis of these comparisons revealed that 822 genes were significantly differentially expressed. Thus, $4 \%$ of the 22,089 Arabidopsis genes represented on the CATMA chip displayed changes in mRNA levels in the plants inoculated with the eH isolate. Among the 822 differentially expressed genes in partial resistance response to $P$. brassicae, 329 were up-regulated whereas 483 were down-regulated. In addition, the expression of ten genes was initially induced, but was then down-regulated (or vice versa) during the time course. A Venn diagram of comparisons 1-3 (Fig. 3) showed that the pattern of host gene expression in partial resistance response became increasingly complex over the time course, with 66, 174, and 706 genes differentially expressed at 1, 2, and $7 \mathrm{dpi}$, respectively.

In order to identify specific transcript changes between partial resistance and susceptibility responses, the transcript profiles of Bur-0 plants inoculated with either the $\mathrm{eH}$ isolate (leading to partial resistance) or with the $\mathrm{e}_{2}$ isolate (leading to complete susceptibility) were compared. Comparisons between the non-inoculated and eH-inoculated plants (described above) showed that host response at the whole-plant level was mainly induced at $7 \mathrm{dpi}$, which corresponds to the initiation of secondary infection in the cortex. Thus, we chose this kinetic point to make comparisons between responses to the $\mathrm{eH}$ and $\mathrm{e}_{2}$ isolates (Fig. 2, comparison 6). Statistical analysis of this comparison revealed 210 genes displaying significant differential expression at $7 \mathrm{dpi}$. Thus, only $1 \%$ of the 22,089 Arabidopsis genes represented on the CATMA chip displayed changes in mRNA levels between susceptible and partial resistance responses. Among these, 120 genes were expressed

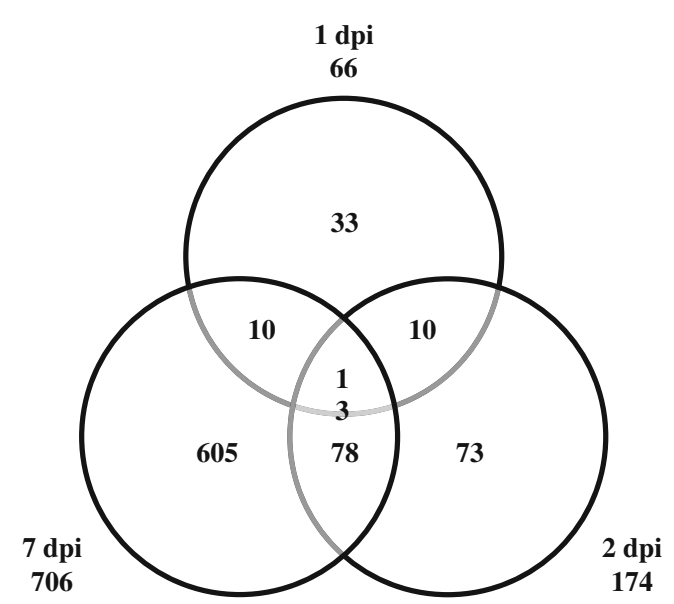

Fig. 3 Venn diagram showing the differential distribution with time point of differentially expressed genes in eH-inoculated plants (partial resistance). $d p i$ days post-inoculation 
Fig. 4 Distribution of the differentially expressed genes classified into functional categories. Genes were assigned to functional categories based on the Functional Catalogue (FunCat) scheme in the MatDB database (MIPS Arabidopsis thaliana Database). Genes of unknown function are not shown. The number of genes identified by the Functional Catalogue is indicated on the $x$-axis

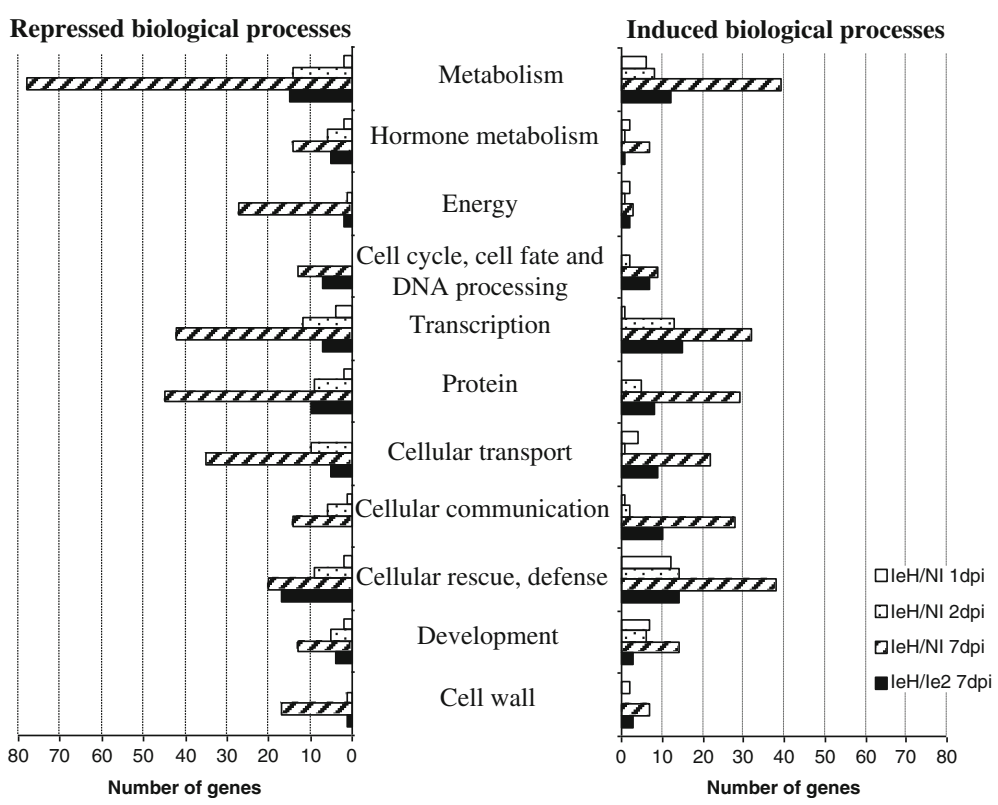

at a higher level in eH-inoculated plants compared to the $\mathrm{e}_{2}$-inoculated ones, whereas 90 genes were expressed at a lower level. Only 94 genes showing differential expression were common to both experiments (comparison between non-inoculated and eH-inoculated plants and comparison between $\mathrm{eH}-$ and $\mathrm{e}_{2}$-inoculated plants).

To investigate which biological processes the differentially regulated genes may be involved in, we classified genes according to the functional categories defined by the Functional Catalogue (FunCat) in the MatDB database (MIPS A. thaliana Database; Fig. 4). This functional categorization carried out on all the genes identified in the time course comparisons between Bur- $0 \mathrm{eH}$-inoculated and control plants showed that genes belonging to all functional groups were affected during the increasing host response to $P$. brassicae infection in the partial resistance response. Furthermore, for almost all biological processes, genes were more often seen to be repressed than induced, with the exception of genes involved in cellular rescue, defense, and cellular communication which were induced. Functional categorization carried out on the genes identified in comparisons between eH- and $\mathrm{e}_{2}$-inoculated plants showed that most of these genes are involved in cellular rescue and defense, metabolism, and transcription-related processes (Fig. 4). A similar number of genes were expressed at a higher or lower level in almost all categories, except for cellular communication and hormone metabolism for which genes were predominantly up- and down-regulated, respectively.

Differential expression in microarrays was confirmed by quantitative RT-PCR

In order to validate the microarray data, a number of genes differentially expressed in at least one of the two studies were selected from different functional categories and their expression measured in control and infected tissues using quantitative RT-PCR. The RT-PCR profiles of these genes revealed that they exhibited the same temporal patterns and direction changes (up- or down-regulated) in gene expression as observed in the microarray experiments (Fig. 5).

The molecular basis of clubroot partial resistance response

We then analyzed the role played by metabolic pathways using the MAPMAN software (Thimm et al. 2004; Fig. 6). MAPMAN is a user-driven tool that displays large data sets, such as gene expression data from Arabidopsis microarrays, onto diagrams of metabolic pathways or other processes in order to highlight general trends. Detailed numeric data are also presented in ESM Tables S2-B to $\mathrm{S} 2-\mathrm{E}$.

\section{Host's primary metabolism regulation in response to eH infection}

Major transcriptional changes in photosynthetic light reactions and carbohydrate metabolism occurred in the partial

Fig. 5 Validation of microarray data by quantitative RT-PCR. Microarray (gray) and quantitative RT-PCR (black) results are expressed in a $\log _{2}$ ratio obtained for comparisons between eH-inoculated $(e H-I)$ and non-inoculated $(N I)$ plants at 1,2 , and $7 \mathrm{dpi}$ for the first experiment and between eH-inoculated $(e H-I)$ and $e_{2}$-inoculated $\left(e_{2}-I\right)$ plants at $7 \mathrm{dpi}$ for the second one. A positive ratio indicates that the gene is significantly induced in eH-inoculated plants in comparison to non-inoculated or $e_{2}$-inoculated plants; a negative ratio means that the gene is significantly repressed in $\mathrm{eH}$-inoculated plants in comparison to noninoculated or $\mathrm{e}_{2}$-inoculated plants. $d p i$ days post-inoculation 


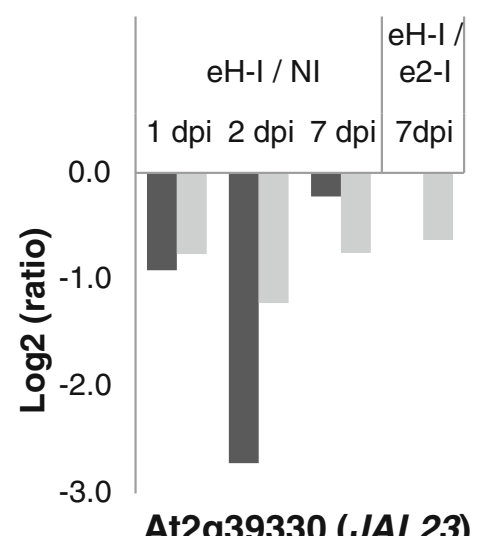

At2g39330 (JAL23)
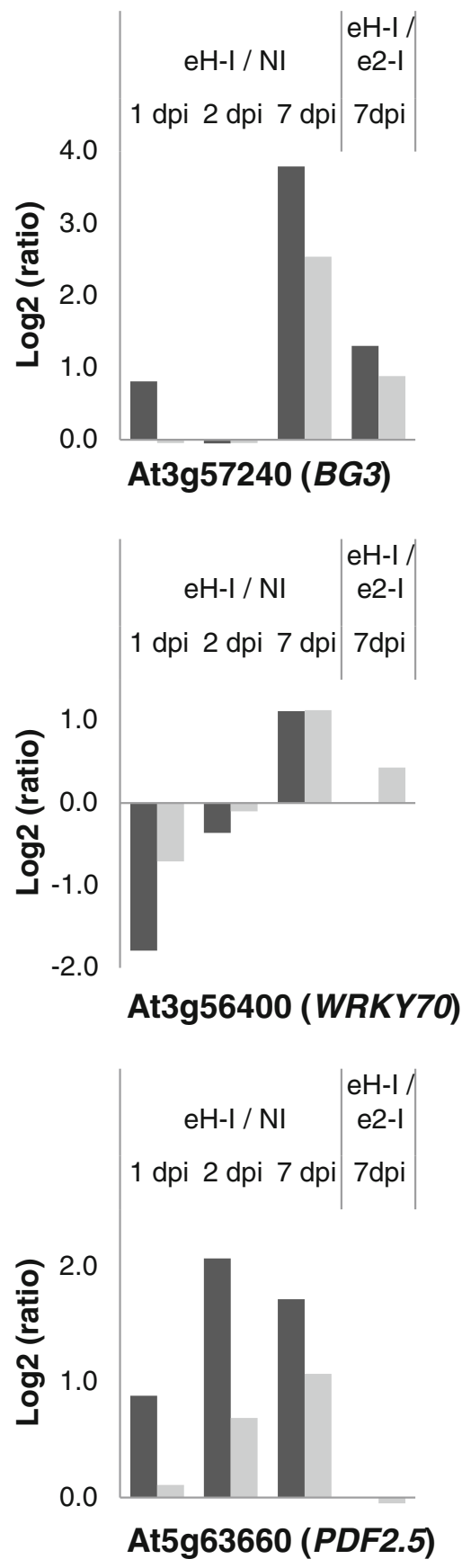
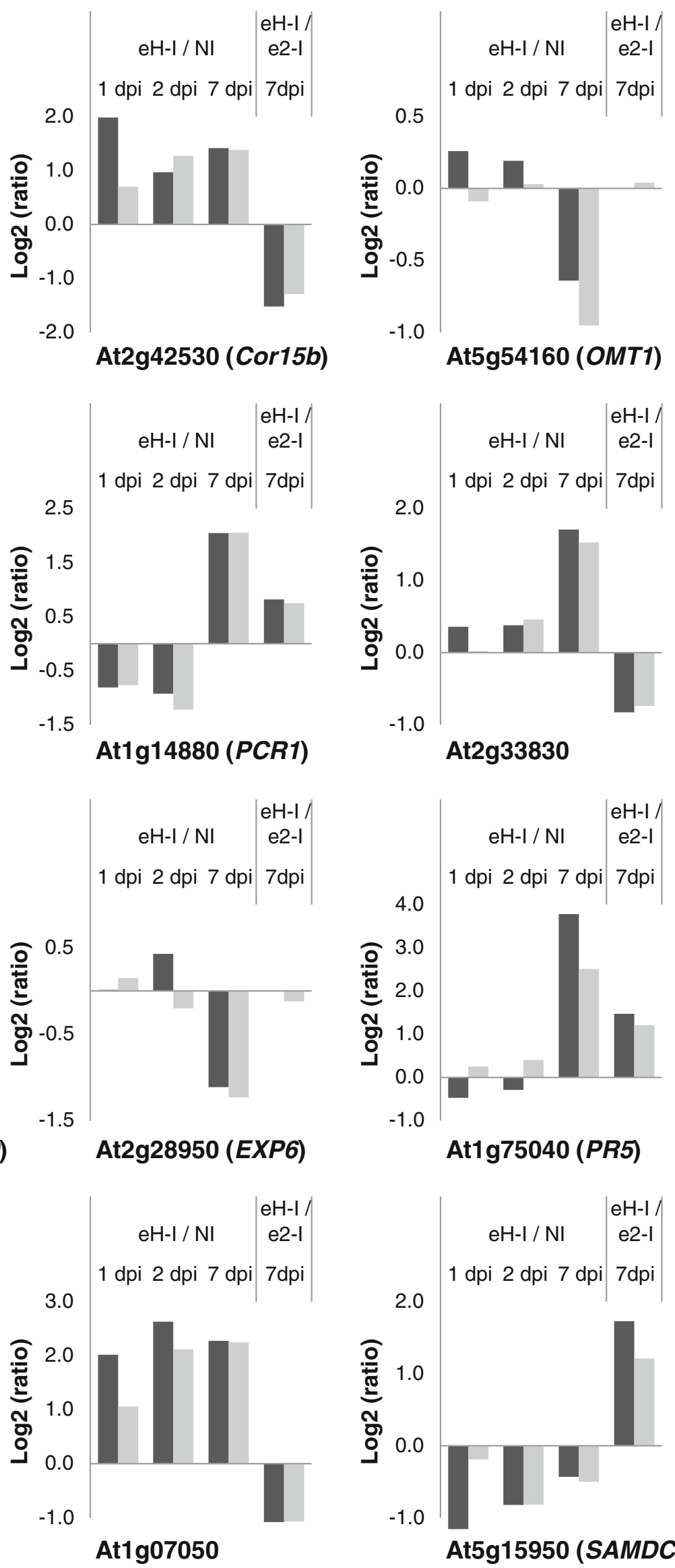


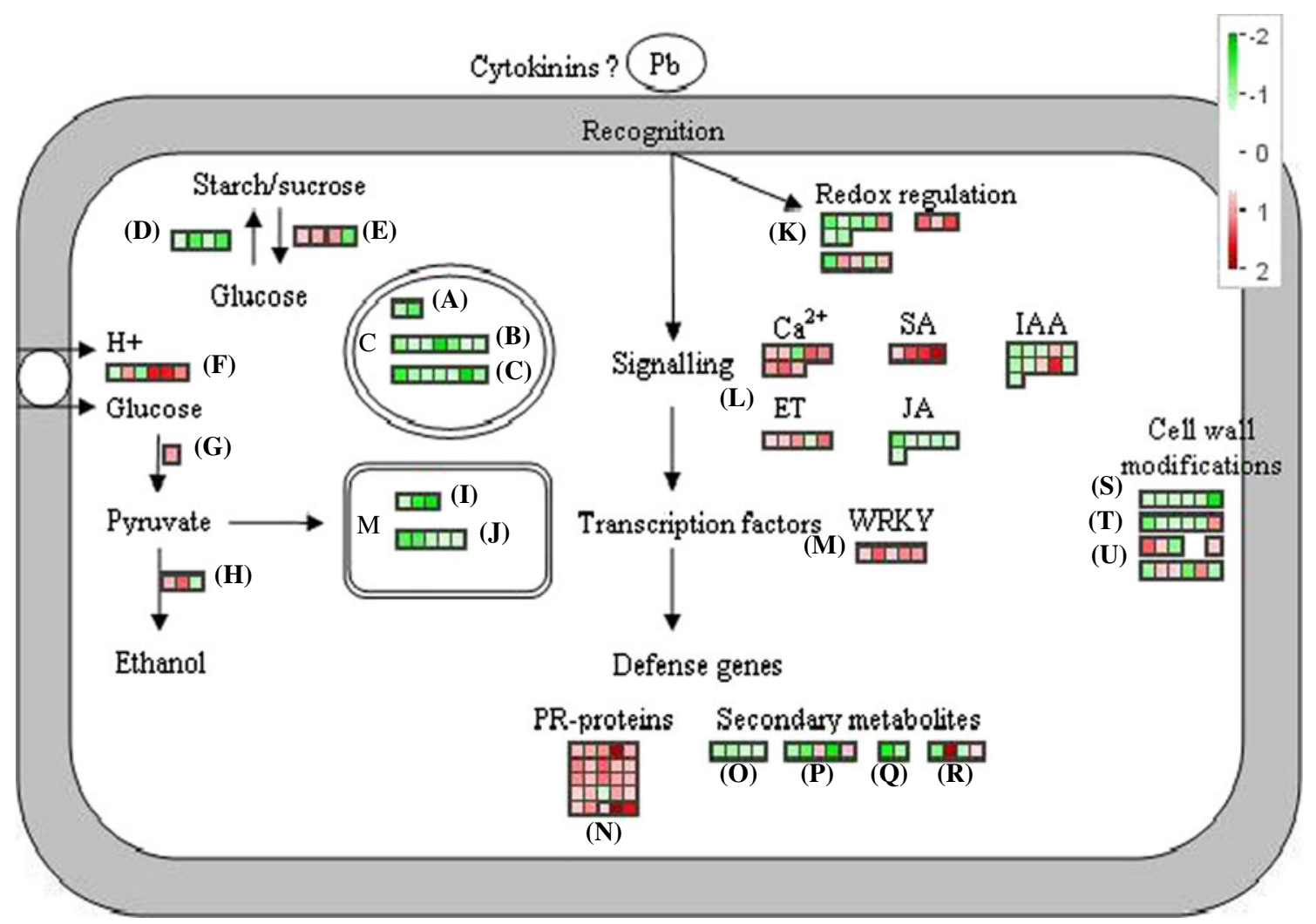

Fig. 6 Schematic representation of the gene expression profiles of metabolic and defense pathways in eH-inoculated plants (partial resistance) at 7 days post-inoculation. Differential gene expression in tetrapyrrole synthesis $(A)$, light reaction enzymes $(B)$, Calvin cycle enzymes $(C)$, starch and sucrose synthesis $(D)$, starch and sucrose degradation $(E)$, sugar transporters $(F)$, glycolysis enzymes $(G)$, fermentation enzymes $(H)$, tricarboxylic acid cycle $(I)$, electron transport enzymes $(J)$, redox status regulation $(K)$, signaling pathways $(L)$, WRKY transcription factors $(M)$, PR proteins $(N)$, isoprenoid metabolism $(O)$, lignin metabolism $(P)$, wax metabolism $(Q)$, flavonoid

resistance response. Indeed, $22 \%$ of all genes differentially expressed in the Bur-0 plants inoculated with the eH isolate were primary metabolism-related genes. The transcript levels of 18 genes involved in tetrapyrrole synthesis and photosynthesis (both the photochemical process and the Calvin cycle) were down-regulated mainly at 7 dpi (Fig. 6 and ESM Table S2-B). Probably linked to this decrease in photosynthesis, the transcript levels of seven genes involved in starch and sucrose synthesis and in the pentose phosphate pathway were also repressed at $7 \mathrm{dpi}$. In contrast, we found that genes coding starch-degrading enzymes, including BAM3 (At4g20270) and PWD (At5g26570), and sucrose-degrading enzymes involved in the biosynthesis of hexose sugars and several sugar transporters were induced in infected plants at $7 \mathrm{dpi}$. In addition, the expression of genes involved in energy production was also altered. Several genes involved in the tricarboxylic acid cycle and in the respiratory chain were repressed. On the contrary, fermentation and production of metabolism $(R)$, cell adhesion $(S)$, cell wall modification $(T)$, and cell wall degradation $(U)$. Each square symbolizes a differentially expressed gene. Numbers +2 to -2 on the color scale represent $\log _{2}$ of the fold change between the inoculated and non-inoculated plants. A positive ratio (red square) indicates that the gene is induced in eHinoculated plants in comparison to non-inoculated plants; a negative ratio (green square) means that the gene is repressed in $\mathrm{eH}$-inoculated plants in comparison to non-inoculated plants. $C$ chloroplast, $M$ mitochondrion, Pb Plasmodiophora brassicae

ethanol appeared to be enhanced as genes encoding pyruvate decarboxylase PDC1 (At5g54960) and PDC2 (At4g33070) were up-regulated.

\section{Transcription factor regulation in response to eH infection}

The expression of many transcription factors and signaling components was altered in response to $P$. brassicae inoculation. Of the overall genes, $13 \%$ were putative transcription factors differentially expressed mainly at $7 \mathrm{dpi}$. These transcription factors belong to several major families, including WRKY, MYB, Basic-Helix-Loop-Helix, Homeobox, and zinc-finger family proteins (Fig. 6 and ESM Table S2-C). Overall, the expression of transcription factors was downregulated, with the exception of the WRKY class which was up-regulated in eH-inoculated plants. The fact that the transcription factors represent a wide distribution of gene families and that different expression profiles were observed may 
suggest that these are involved in controlling different processes and/or different phases of the response.

\section{Host defense responses to eH infection}

Further analysis showed that most of the 822 clubrootinduced Arabidopsis genes are potentially involved in host defense responses. In addition to WRKY transcription factors, some other signaling components, such as the calcium signaling pathway, were differentially expressed (Fig. 6 and ESM Table S2-D). Whereas two genes encoding calmodulins and calcium-binding proteins were down-regulated at the first two time points, seven were up-regulated at $7 \mathrm{dpi}$. Lastly, genes known or predicted to encode kinases were particularly abundant among the clubroot-induced genes. Numerous known or putative kinases were induced by $P$. brassicae inoculation at the end of the time course analysis. Of particular interest were 12 leucin-rich repeat (LRR)-containing receptor-like kinases. In contrast, four genes encoding G-proteins were down-regulated at $7 \mathrm{dpi}$.

The third largest functional class (12\%) contained genes involved in cell rescue and defense. Two thirds of these genes were up-regulated. At the first two time points postinoculation, the defense-related group mainly included genes that were previously shown to be induced during abiotic stress, such as heat shock proteins or dehydrins. In contrast, at the later time point, the defense-related group was predominantly composed of genes encoding proteins frequently described during plant-pathogen interactions, such as enzymes involved in the removal of reactive oxygen species (ascorbate peroxidases, glutathione peroxidase, peroxidases, superoxide dismutase, glutathione-S-transferases) or pathogenesis-related (PR) proteins such as $\beta$-1,3-glucanases, chitinases, thaumatin, and defensins.

The transcript levels of some genes involved in secondary metabolism were also modulated in the partial resistance response. Two branches of the phenylpropanoid pathway were altered upon eH inoculation. In the flavonoid biosynthesis pathway, flavonol production appeared to have been favored since the gene encoding the flavonol synthase (At5g08640) was slightly up-regulated from 2 dpi. On the contrary, a gene encoding dihydroflavonol-4reductase (At4g27250), which drives the same substrate (dihydroflavonol) into another pathway leading to anthocyanins and tannins, was down-regulated at $7 \mathrm{dpi}$. Among the flavonoid-related gene subset, DMR6 (At5g24530) exhibited the highest induction level at $7 \mathrm{dpi}$. This gene, encoding an enzyme whose exact substrate is suspected to be a flavonoid, is known to be induced by the salicylic acid analogue BTH treatments and was reported to be involved in resistance to Hyaloperonospora parasitica (van Damme et al. 2008). Flavonoid accumulation was also reported in Arabidopsis roots following P. brassicae infection and was associated with the modulation of auxin efflux (Pasold et al. 2010). The lignin biosynthesis pathway was also affected; however, because genes involved in the same biosynthesis step showed conflicting differential regulations, the physiological impact of this pathway is unclear. Lastly, the expression of genes involved in isoprenoid and wax biosyntheses were down-regulated.

Analysis of the data also indicated that $P$. brassicae challenge significantly targeted several plant hormone signaling and stress response pathways. The down-regulation of genes encoding proteins involved in jasmonic acid (JA) biosynthesis, such as the lipoxygenase LOX2 (At3g45140), in the JA signaling pathway, such as the desaturase SSI2 (At2g43710), or jasmonate-inducible genes such as beta-glucosidase $B G 1$ (Atlg52400), jacalin lectin proteins (At3g16450, At3g16470) , hydroperoxide lyase HPL1 (At4g15440), and the GH3 family member JAR1 (At2g46370) suggested a down-regulation of the JA pathway at 2 and 7 dpi. The gene encoding WRKY70 (At3g56400), a transcription factor which is well known to suppress jasmonic acid responses ( $\mathrm{Li}$ et al. 2006), was upregulated at $7 \mathrm{dpi}$. Interestingly, in contrast, three ethylene (ET)-related genes involved in either synthesis, such as 1aminocyclopropane-1-carboxylic acid (ACC) synthase (At1g03400) and ACC oxidase (At5g43440), or response were up-regulated at 7 dpi.

Several genes related to the acid salicylic (SA) pathway were also induced, such as the isochorismate synthase ICSI (At1g74710) involved in SA biosynthesis and the lipase-like protein PAD4 (At3g52430) and two NPR1-interacting proteins-NIMIN1 (At1g02450) and NIMIN2 (At3g25882)components of this defense signaling pathway. Furthermore, the genes SSI2 (At2g43710) and JAR1 (At2g46370), previously shown to negatively interact with the SA pathway (Thatcher et al. 2005), were down-regulated.

\section{Cell division and expansion regulation in response to eH infection}

Several genes involved in growth and cell cycle control, including cellular organization and cell division, were also differentially expressed, mainly at 7 dpi (Fig. 6 and ESM Table S2-E). Furthermore, three members of fasciclin-like arabinogalactan proteins, involved in cell adhesion, were also repressed at 7 dpi. Numerous genes involved in cell wall modification were differentially expressed in the plants inoculated with the isolate eH, such as expansins and xyloglucan endotransglycosylases, which were down-regulated from 2 dpi. Pectinesterases were mainly up-regulated at 7 dpi. Genes involved in cell wall degradation such as $\beta$ 1,4-endoglucanase, glycosyl hydrolases, polygalacturonase, and pectate lyase were also differentially expressed.

$P$. brassicae inoculation modulated the stress hormone response pathway (JA, ET, SA), but plant host physiology 
also experienced specific shifts in auxin and cytokinin responses as well. Twelve auxin-related genes were differentially expressed following infection by $P$. brassicae isolate eH. Several auxin-related genes were differentially expressed at $1 \mathrm{dpi}$, and their number increased with the infection. These include genes involved in auxin synthesis, such as nitrilase NIT1 (At3g44310) and myrosinase-binding proteins (At2g39310, At2g39330), as well as auxin transport and response, and these were mostly down-regulated. Only three auxin-related genes were up-regulated at $7 \mathrm{dpi}$. Two genes involved in cytokinin metabolism were repressed at 7 dpi: the isopentenyltransferase IPT9 (At5g20040), involved in cytokinin biosynthesis, and the response regulator ARR4 (Atlg10470).

What are the differences between partial clubroot resistance and susceptibility responses?

\section{Host's primary metabolism regulation}

Thirteen percent of all the genes that are differentially expressed between $\mathrm{eH}$ and $\mathrm{e}_{2}$ responses are predicted to function in primary metabolism. Almost all of them were found to be $\mathrm{e}_{2}$-specific or $\mathrm{e}_{2}$-enhanced regulations (ESM Table S2-B). As reported above, several genes involved in photosynthesis were repressed by eH at $7 \mathrm{dpi}$. Among those, the gene GUN4 (At3g59400) involved in tetrapyrrole biosynthesis was found to be more repressed by $\mathrm{e}_{2}$ than by $\mathrm{eH}$, and four other genes (At5g24120, At5g13630, At1g58290, and At2g21330) involved in tetrapyrrole biosynthesis, photosynthesis regulation, and Calvin cycle were specifically repressed by $\mathrm{e}_{2}$. Similarly, the $\mathrm{e}_{2}$-specific induction of $D P E$ (At2g40840) and the $\mathrm{e}_{2}$-enhanced induction of $P W D$ (At5g26570), both involved in starch degradation, may suggest that $\mathrm{e}_{2}$ infection could result in an enhanced starch degradation process. The level of induction of the gene encoding the pyruvate decarboxylase PDC1 (At5g54960) was lower in the eH response, suggesting that the metabolic shift from aerobic to anaerobic fermentation associated with $P$. brassicae inoculation reported in the first microarray comparisons was also less pronounced in the partial resistant response than in the susceptible one.

\section{Transcription factor regulation}

Several putative transcription factors, representing almost $11 \%$ of the differentially expressed genes, showed differential expression depending on response type. Seven transcription factors belonging to the WRKY, MYB, bZIP, and zinc finger families were specifically repressed by $e_{2}$ infection, and two were specifically induced by eH infection (WRK46 and At5g10380 coding a C3HC4-type RING finger protein).

\section{Host defense responses}

Compared to the gene expression patterns observed in response to $\mathrm{eH}$, defense responses upon inoculation with the more aggressive isolate $e_{2}$ were lower. Six percent of the genes which were differentially expressed between $\mathrm{eH}$ - and $\mathrm{e}_{2}$-inoculated plants are involved in signal transduction. Three genes coding kinases and three genes coding calmodulin-binding proteins were repressed specifically by infection with the isolate $e_{2}$. Two genes coding protein kinase were also induced specifically by $e_{2}$. One gene coding a calmodulin, one DUF receptor kinase, and one LRR-containing receptor kinase were specifically induced by $\mathrm{eH}$. The second largest functional class $(15 \%)$ contained genes involved in cell rescue and defense. Approximately half of these genes were previously shown to be induced during abiotic stress and were mainly induced in the $e_{2}$-inoculated plants. The others have been frequently described during plant-pathogen interactions, such as genes involved in the removal of reactive oxygen species (glutathione peroxidase, peroxidase, catalases, and glutathione-Stransferase) or genes encoding pathogenesis-related proteins (thaumatin and defensin). Few genes involved in secondary metabolism such as the phenylpropanoid and the isoprenoid biosynthesis pathways were differentially expressed between $\mathrm{eH}$ and $\mathrm{e}_{2}$ responses. Two genes involved in the phenylpropanoid pathway displayed $\mathrm{e}_{2}$-specific or $\mathrm{e}_{2}$-enhanced induction. The transcript levels of the genes At1g06570 and At4g32770 encoding 4-hydroxyphenylpyruvate dioxygenase and tocopherol cyclase, respectively, were specifically repressed in response to $e_{2}$, suggesting that this regulation might be involved in susceptibility. Lastly, the repression of the CERI gene (At1g022050) involved in wax biosynthesis was enhanced in response to $\mathrm{eH}$, suggesting that this regulation could be involved in partial resistance.

At 7 dpi, several JA-related genes were found to be specifically induced by $\mathrm{e}_{2}$ (ESM Table S2-D and Fig. S3), such as the JA biosynthetic genes LOX2 (At3g45140), encoding lipoxygenase, and AOS (At5g46250), encoding allene oxide synthase, and the JA-inducible genes BG1 (At1g52400) encoding a beta-glucosidase and CYP81D1 (At3g28740). The induction of the SA pathway appeared to be enhanced in eH response as the lipase-like protein PAD4 (At3g52430) and the NPR1-interacting protein NIMIN2 (At3g25882) inductions were found to be clearly specific or enhanced in response to $\mathrm{eH}$. $\mathrm{e}_{2}$-specific repression, at the transcriptional level of few ethylene-related genes in at least one replicate (At5g25190, At5g61590, At5g47220, and At2g27050) may suggest also an enhanced ET pathway in $\mathrm{eH}$ response.

\section{Cell division and expansion regulation}

Four genes involved in cell growth and cycle control display $\mathrm{e}_{2}$-specific or $\mathrm{e}_{2}$-enhanced induction: the annexin $A N N 4$ 
(At2g38750), the expansin EXP16 (At3g55500), a caldesmon-related protein (At1g52410), and a nodulin $\mathrm{mtN} 3$ family protein (At5g23660). The genes At4g02330 encoding a putative pectinesterase and FLA9 (At1g03870) encoding a fasciclin-like arabinogalactan protein were specifically repressed in the $\mathrm{e}_{2}$ response. The gene SEN4 (At4g30270) encoding an endo-xyloglucan endo1,4- $\beta$ glucanase was more repressed by $\mathrm{e}_{2}$ than by $\mathrm{eH}$.

Lastly, several auxin-related genes were differentially affected by $\mathrm{eH}$ and $\mathrm{e}_{2}$ isolates in at least one replicate. These include genes involved in auxin biosynthesis, such as nitrilase NIT1 (At3g44310) and myrosinase-binding proteins (Atlg52000, At2g39330), which were more repressed in response to $\mathrm{eH}$ than to $\mathrm{e}_{2}$. Genes involved in auxin response (At1g16510, At2g33830) were also repressed in response to the eH isolate. In particular, the gene At2g33830 encoding a dormancy/auxin-associated protein, previously reported as induced by eH inoculation, showed lower expression in response to $\mathrm{eH}$ than to $\mathrm{e}_{2}$.

\section{Discussion}

The identification of genes regulated in partial clubroot resistance responses represents a major challenge for understanding the basis of partial quantitative resistance. Genome-wide comparative transcriptional analyses revealed here major differential gene expressions including a reduced or delayed metabolic diversion by the pathogen, an earlier and/or stronger induction of classical defense responses, and an active inhibition of cell enlargement and proliferation in the clubroot partial resistance response compared to the susceptible one.

A relatively small proportion of genes displayed significant changes in expression during the partial resistance response to the $P$. brassicae isolate $\mathrm{eH}$. This result is in agreement with the earlier transcriptomics or proteomics works carried out at the early stages of host-pathogen interaction on fully susceptible Brassicaceae accessions (Agarwal et al. 2011; Devos et al. 2006; Cao et al. 2008) and contrasts with the results of Siemens et al. (2006) who reported high numbers of differentially expressed genes at greater fold change during the second stage of the disease. Together, these results suggest that during the asymptomatic phase of the $P$. brassicae life cycle, fewer morphological and physiological changes occur in the host compared with the secondary stage, when the host roots exhibit growing galls. Expression pattern comparisons between noninoculated and eH-inoculated plants revealed an initial host response from $1 \mathrm{dpi}$ that became increasingly complex. Relatively few genes were differentially expressed at 1 and $2 \mathrm{dpi}$, during the first contact between primary zoospores and root hairs and the development of primary plasmodia, and most were components of a general stress response.
Most of the metabolic changes and defense systems specific to pathogen response were only triggered at $7 \mathrm{dpi}$, when secondary infection in the cortex is initiated. This increasing response pattern sharply contrasts with the decreasing host response to infection by $P$. brassicae observed in a susceptible Arabidopsis accession by Agarwal et al. (2011) and, thus, could be a key component of partial resistance.

The pathogenesis process leads to reprogramming of the host's primary metabolism

First, this study showed that upon inoculation with $P$. brassicae, the host's primary metabolism underwent major reprogramming (Fig. 6), in particular with the repression of genes involved in the photochemical processes of photosynthesis and the Calvin cycle in infected plants, suggesting a low de novo carbohydrate production in leaves. This is rather coherent with the model proposed by Devos et al. (2006) where a leaf growth decrease coincides with the beginning of secondary infection. Together with the concomitant repression of genes involved in starch biosynthesis and the induction of genes involved in starch degradation and sugar transport (Fig. 6), those data are consistent with the accepted model where leaf carbon assimilates are reallocated to the infected root sink (Keen and Williams 1969; Evans and Scholes 1995). This flow toward the production of glucose was previously suggested in the proteome and transcriptome analyses of the susceptible ecotype Col-0 upon P. brassicae infection (Devos et al. 2006; Siemens et al. 2006). Furthermore, the suppression of invertase activity, which hydrolyzes sucrose into hexose monomers, using transgenic Arabidopsis lines leads to clearly reduced clubroot symptoms (Siemens et al. 2011).

Energy production mechanisms were also altered upon $P$. brassicae inoculation with the repression of cellular respiration processes and the induction of glycolysis and ethanol fermentation (Fig. 6). The infection appears to have induced a metabolic shift at $7 \mathrm{dpi}$ from aerobic to anaerobic fermentation. This switch was also observed in the A. thalianaAgrobacterium tumefasciens interaction (Deeken et al. 2006). As a result of diffusional limitations due to gall formation, cells in the infected tissues may easily become hypoxic and switch to fermentative energy metabolism. A second hypothesis was suggested by Koch et al. (2000) who showed that alcohol dehydrogenase $A D H 1$, a main regulatory enzyme of ethanol fermentation, responds to sugars at physiological concentrations in fully oxygenated maize root tips. The accumulation of sugars at the infection sites might thus induce a hypoxia-like response and consequently force plant cells to switch to fermentative energy metabolism.

These metabolic changes occurred in both susceptible and partial resistance responses. However, several gene regulations related to photosynthesis and starch degradation 
suggest that the consequences on host primary metabolism were more dramatic in the susceptible response than in the partial resistance one. Consistent with these observations, Wagner et al. (2012) showed in B. napus that the primary metabolism of resistant genotypes was not much affected compared to susceptible ones in response to P. brassicae infection. Hence, the partial resistance response may be associated with a reduced or delayed metabolic diversion by the pathogen. However, we cannot conclude yet whether this regulation constitutes the cause, by retarding plasmodia growth in host cells, or the result of partial clubroot resistance.

Defense responses were induced earlier or at increased levels during the partial resistance response

The identification of genes differentially expressed in the partial resistance response to $P$. brassicae demonstrated the activation of defense responses common to the basal defense and $R$-mediated resistance responses (HammondKosack and Parker 2003; Fig. 6). Moreover, a comparison of $\mathrm{eH}$ and $\mathrm{e}_{2}$ responses at 7 dpi showed that several genes involved in defense mechanisms specific to the pathogen infection response were activated or induced at a higher level in response to eH (i.e., partial resistance response). In response to the $\mathrm{e}_{2}$ isolate (i.e., susceptibility response), they were either not induced at all or induced at significantly lower levels. Instead, response to $\mathrm{e}_{2}$ included a specific or enhanced activation of the components of the general stress response, such as those observed in eH-inoculated plants at 1 and 2 dpi. In agreement with these results, both Agarwal et al. (2011) and Siemens et al. (2006) reported that during the infection of a susceptible Arabidopsis accession by P. brassicae, few defense and disease resistance responses were activated or were even strongly down-regulated. It is consequently tempting to speculate, by analogy to the proposed hypothesis for incompatible interactions, that stronger or earlier signaling events enable eH-inoculated plants to delay and/or attenuate the effects of virulence factors (Tao et al. 2003; Poland et al. 2009).

Following pathogen attack, early defense signaling events are amplified through the generation of secondary signaling molecules, such as SA, JA, and ET, which activate defenses both locally, at the site of infection, and systematically in non-infected tissues. We found here that both the SA and ET pathways were induced during the partial resistance response, whereas the JA pathway was repressed (Fig. 6). The involvement of the SA, JA, or the ET pathway in the Brassicaceae-P. brassicae pathosystem has been previously described. In Chinese cabbage, a highly susceptible host. JA levels increased during club development and may be involved in the up-regulation of nitrilase, myrosinase, and tryptophan oxidase, enzymes involved in IAA synthesis
(Grsic et al. 1999). Variations in the concentration of ACC, the direct precursor of ET, were observed in infected Chinese cabbage roots during the initiation of secondary infection (Devos et al. 2005). Further evidence for the involvement of the ET and JA pathways in gall formation was also provided by mutant analyses (Siemens et al. 2002, 2006; Devos et al. 2006). In addition, transcriptomic analyses on the Arabidopsis susceptible accession Col-0 at the earlier and later stages of the infection showed the induction of the JA pathway and the repression of the SA and ET pathways (Siemens et al. 2006; Agarwal et al. 2011). Pretreatment of Arabidopsis plants with salicylic acid was able to reduce the susceptibility to $P$. brassicae (Agarwal et al. 2011). This is consistent with our results where repression of the JA pathway and induction of the ET and SA pathways were associated with a reduction in symptom severity.

Partial resistance is associated with an inhibition of cell division and expansion

Lastly, we identified several genes which could be involved in clubroot-induced uncontrolled cell division and expansion, such as genes controlling cytoskeleton dynamics, cell adhesion, and cell wall modifications (Fig. 6). As expected, most of these genes were differentially expressed at $7 \mathrm{dpi}$, when secondary infection in the cortex is initiated. Furthermore, most expansins were also down-regulated at $7 \mathrm{dpi}$, which would consequently lead to reduced galls.

The intrinsic characteristics of clubroot disease point to an involvement of the plant hormones cytokinin and auxin. A role for cytokinins (Dekhuijzen and Overeem 1971; Devos et al. 2005; Muller and Hilgenberg 1986; Siemens et al. 2006) and auxins was established in gall development (Grsic et al. 1999; Ludwig-Muller et al. 1993, 1999, 2009; Neuhaus et al. 2000) as well as in the early stage of infection (Devos et al. 2006; Agarwal et al. 2011). The current study also supports a possible role for cytokinins in early infection events with the repression of cytokinin biosynthesis upon $P$. brassicae infection. Furthermore, consistent with our microarray results carried out on partial resistance response, transgenic plants with lower cytokinin levels were found to be more tolerant to clubroot (Siemens et al. 2006). Host auxin metabolism is redirected toward the synthesis of more auxin through the nitrilase pathway (Grsic-Rausch et al. 2000) as clubroot plasmodia act as a strong sink for auxins. Siemens et al. (2006) reported the expression induction of the genes encoding nitrilases 1 and 2 during club development in the susceptible accession Col-0. Moreover, Devos et al. (2006) showed, at the proteome level, the upregulation of myrosinase and myrosinase-binding protein at $4 \mathrm{dpi}$ in the susceptible accession Col-0. In B. rapa, the level of expression of myrosinase increased in infected roots compared with 
controls (Grsic et al. 1999). In our microarray analysis, numerous genes involved in host auxin biosynthesis as well as auxin transport and response were down-regulated in $\mathrm{eH}-$ inoculated plants (Fig. 6). The down-regulation of this pathway is consistent with the reduced gall formation in the partial resistant response. In agreement with this result, a nitrilase 1 mutant nitl was shown to have reduced root gall size and lower free IAA content in clubs (Grsic-Rausch et al. 2000; Neuhaus et al. 2000), and transgenic plants reduced in nitrilase 2 showed slower development of root galls (Neuhaus et al. 2000). Consequently, differential regulation of metabolic pathways related to the development of clubroot symptoms in eH-inoculated plants is consistent with reduced gall formation and suggests the existence of an as yet unknown mechanism associated with the reduction of cell enlargement and proliferation in the partial resistance response.

Further work on these pathways, such as their genetic manipulation in A. thaliana transgenic plants or quantification of gene expression in a range of Arabidopsis and Brassicas, showing extreme and intermediate levels of resistance to clubroot, will provide insights into the mechanisms involved in partial clubroot resistance.

Acknowledgments We acknowledge our colleagues of OUESTGénopole ${ }^{\circledR}$ for technical assistance. This work was supported by the French National Institute for Agronomic Research (INRA). Mélanie Jubault was a PhD student funded by the French Ministry of Research.

Open Access This article is distributed under the terms of the Creative Commons Attribution License which permits any use, distribution, and reproduction in any medium, provided the original author(s) and the source are credited.

\section{References}

AbuQamar S, Chen X, Dhawan R, Bluhm B, Salmeron J, Lam S, Dietrich RA, Mengiste T (2006) Expression profiling and mutant analysis reveals complex regulatory networks involved in Arabidopsis response to Botrytis infection. Plant $\mathrm{J}$ 48(1):28-44

Agarwal A, Kaul V, Faggian R, Rookes JE, Ludwig-Muller J, Cahill DM (2011) Analysis of global host gene expression during the primary phase of the Arabidopsis thaliana-Plasmodiophora brassicae interaction. Funct Plant Biol 38(6):462-478

Alix K, Lariagon C, Delourme R, Manzanares-Dauleux MJ (2007) Exploiting natural genetic diversity and mutant resources of Arabidopsis thaliana to study the A. thaliana-Plasmodiophora brassicae interaction. Plant Breed 126(2):218-221

Allemeersch J, Durinck S, Vanderhaeghen R, Alard P, Maes R, Seeuws K, Bogaert T, Coddens K, Deschouwer K, Van Hummelen P, Vuylsteke M, Moreau Y, Kwekkeboom J, Wijfjes AHM, May S, Beynon J, Hilson P, Kuiper MTR (2005) Benchmarking the CATMA microarray. A novel tool for Arabidopsis transcriptome analysis. Plant Physiol 137(2):588-601

Boyd LA (2006) Can the durability of resistance be predicted? J Sci Food Agric 86(15):2523-2526

Boyes DC, Zayed AM, Ascenzi R, McCaskill AJ, Hoffman NE, Davis KR, Gorlach J (2001) Growth stage-based phenotypic analysis of
Arabidopsis: a model for high throughput functional genomics in plants. Plant Cell 13(7):1499-1510

Brun H, Chevre AM, Fitt BDL, Powers S, Besnard AL, Ermel M, Huteau V, Marquer B, Eber F, Renard M, Andrivon D (2010) Quantitative resistance increases the durability of qualitative resistance to Leptosphaeria maculans in Brassica napus. New Phytol 185(1):285-299

Cao T, Srivastava S, Rahman MH, Kav NNV, Hotte N, Deyholos MK, Strelkov SE (2008) Proteome-level changes in the roots of Brassica napus as a result of Plasmodiophora brassicae infection. Plant Sci 174(1):97-115

Crowe ML, Serizet C, Thareau V, Aubourg S, Rouze P, Hilson P, Beynon J, Weisbeek P, Hummelen P, Reymond P, Paz-Ares J, Nietfeld W, Trick M (2003) CATMA: a complete Arabidopsis GST database. Nucleic Acids Res 31(1):156-158

de Jong M, van Breukelen B, Wittink FR, Menke FLH, Weisbeek PJ, Van den Ackerveken G (2006) Membrane-associated transcripts in Arabidopsis; their isolation and characterization by DNA microarray analysis and bioinformatics. Plant J 46(4):708-721

Deeken R, Engelmann JC, Efetova M, Czirjak T, Muller T, Kaiser WM, Tietz O, Krischke M, Mueller MJ, Palme K, Dandekar T, Hedrich R (2006) An integrated view of gene expression and solute profiles of Arabidopsis tumors: a genome-wide approach. Plant Cell 18(12):3617-3634

Dekhuijzen HM, Overeem JC (1971) The role of cytokinins in clubroot formation. Physiol Plant Pathol 1:151-161

Devos S, Vissenberg K, Verbelen JP, Prinsen E (2005) Infection of Chinese cabbage by Plasmodiophora brassicae leads to a stimulation of plant growth: impacts on cell wall metabolism and hormone balance. New Phytol 166(1):241-250

Devos S, Laukens K, Deckers P, Straeten DV, Beeckman T, Inze D, Onckelen H, Witters E, Prinsen E (2006) A hormone and proteome approach to picturing the initial metabolic events during Plasmodiophora brassicae infection on Arabidopsis. Mol Plant Microbe Interact 19(12):1431-1443

Diederichsen E, Frauen M, Linders EGA, Hatakeyama K, Hirai M (2009) Status and perspectives of clubroot resistance breeding in Crucifer crops. J Plant Growth Regul 28 (3):265-281

Ditt RF, Kerr KF, Figueiredo P, Delrow J, Comai L, Nester EW (2006) The Arabidopsis thaliana transcriptome in response to Agrobacterium tumefaciens. Mol Plant Microbe Interact 19(6):665-681

Dixon GR (2009) The occurrence and economic impact of Plasmodiophora brassicae and clubroot disease. J Plant Growth Regul 28(3):194-202

Donald C, Porter I (2009) Integrated control of clubroot. J Plant Growth Regul 28(3):289-303

Evans JL, Scholes JD (1995) How does clubroot alter the regulation of carbon metabolism in its host? Asp Appl Biol 42:125-132

Fähling M, Graf H, Siemens J (2003) Pathotype separation of Plasmodiophora brassicae by the host plant. J Phytopathol Phytopathol Z 151(7-8):425-430

Gravot A, Grillet L, Wagner G, Jubault M, Lariagon C, Baron C, Deleu C, Delourme R, Bouchereau A, Manzanares-Dauleux MJ (2011) Genetic and physiological analysis of the relationship between partial resistance to clubroot and tolerance to trehalose in Arabidopsis thaliana. New Phytol 191(4):1083-1094

Gravot A, Deleu C, Wagner G, Lariagon C, Lugan R, Todd C, Wendehenne D, Delourme R, Bouchereau A, ManzanaresDauleux MJ (2012) Arginase induction represses gall development during clubroot infection in Arabidopsis. Plant Cell Physiol 53(3):901-911

Grsic S, Kirchheim B, Pieper K, Fritsch M, Hilgenberg W, LudwigMuller J (1999) Induction of auxin biosynthetic enzymes by jasmonic acid and in clubroot diseased Chinese cabbage plants. Physiol Plant 105(3):521-531 
Grsic-Rausch S, Kobelt P, Siemens JM, Bischoff M, Ludwig-Muller J (2000) Expression and localization of nitrilase during symptom development of the clubroot disease in Arabidopsis. Plant Physiol 122(2):369-378

Hammond-Kosack KE, Parker JE (2003) Deciphering plant-pathogen communication: fresh perspectives for molecular resistance breeding. Curr Opin Biotechnol 14(2):177-193

Hilson P, Allemeersch J, Altmann T, Aubourg S, Avon A, Beynon J, Bhalerao RP, Bitton F, Caboche M, Cannoot B, Chardakov V, Cognet-Holliger C, Colot V, Crowe M, Darimont C, Durinck S, Eickhoff $\mathrm{H}$, de Longevialle AF, Farmer EE, Grant M, Kuiper MTR, Lehrach H, Leon C, Leyva A, Lundeberg J, Lurin C, Moreau Y, Nietfeld W, Paz-Ares J, Reymond P, Rouze P, Sandberg G, Segura MD, Serizet C, Tabrett A, Taconnat L, Thareau V, Van Hummelen P, Vercruysse S, Vuylsteke M, Weingartner M, Weisbeek PJ, Wirta V, Wittink FRA, Zabeau M, Small I (2004) Versatile gene-specific sequence tags for Arabidopsis functional genomics: transcript profiling and reverse genetics applications. Genome Res 14(10B):2176-2189

Huibers RP, de Jong M, Dekter RW, Van den Ackerveken G (2009) Disease-specific expression of host genes during downy mildew infection of Arabidopsis. Mol Plant-Microbe Interact 22 (9):1104-1115

Ingram DS, Tommerup IC (1972) The life history of Plasmodiophora brassicae Woron. Proc R Soc London, Ser B 180(1058):103-112

Jammes F, Lecomte P, Almeida-Engler J, Bitton F, Martin-Magniette M-L, Renou JP, Abad P, Favery B (2005) Genome-wide expression profiling of the host response to root-knot nematode infection in Arabidopsis. Plant J 44(3):447-458

Jubault M, Hamon C, Gravot A, Lariagon C, Delourme R, Bouchereau A, Manzanares MJ (2008a) Differential regulation of root arginine catabolism and polyamine metabolism in clubroot-susceptible and partially resistant Arabidopsis genotypes. Plant Physiol 146 (4):2008-2019

Jubault M, Lariagon C, Simon M, Delourme R, Manzanares-Dauleux MJ (2008b) Identification of quantitative trait loci controlling partial clubroot resistance in new mapping populations of Arabidopsis thaliana. Theor Appl Genet 117(2):191-202

Keen NT, Williams PH (1969) Translocation of sugars into infected cabbage tissues during clubroot development. Plant Physiol 44 (5):748-754

Koch KE, Ying Z, Wu Y, Avigne WT (2000) Multiple paths of sugarsensing and a sugar/oxygen overlap for genes of sucrose and ethanol metabolism. J Exp Bot 51:417-427

Li J, Brader G, Kariola T, Palva ET (2006) WRKY70 modulates the selection of signaling pathways in plant defense. Plant J 46 (3):477-491

Ludwig-Muller J, Bendel U, Thermann P, Ruppel M, Epstein E, Hilgenberg W (1993) Concentrations of indole-3-acetic acid in plants of tolerant and susceptible varieties of Chinese cabbage infected with Plasmodiophora brassicae Woron. New Phytol 125 (4):763-769

Ludwig-Muller J, Bennett RN, Kiddle G, Ihmig S, Ruppel M, Hilgenberg W (1999) The host range of Plasmodiophora brassi$c a e$ and its relationship to endogenous glucosinolate content. New Phytol 141(3):443-458

Ludwig-Muller J, Prinsen E, Rolfe SA, Scholes JD (2009) Metabolism and plant hormone action during clubroot disease. J Plant Growth Regul 28(3):229-244

Manzanares-Dauleux MJ, Delourme R, Baron F, Thomas G (2000a) Mapping of one major gene and of QTLs involved in resistance to clubroot in Brassica napus. Theor Appl Genet 101(5/6):885891

Manzanares-Dauleux MJ, Divaret I, Baron F, Thomas G (2000b) Evaluation of French Brassica oleracea landraces for resistance to Plasmodiophora brassicae. Euphytica 113(3):211-218
Manzanares-Dauleux MJ, Delourme R, Glory P, Giboulot A, Thomas G (2003) Mapping QTLs and major resistance genes to clubroot (Plasmodiophora brassicae) in Brassica napus. 13th Crucifer Genetics Workshop, UC Davis, California, March 23-26

Marathe R, Guan Z, Anandalakshmi R, Zhao HY, Dinesh-Kumar SP (2004) Study of Arabidopsis thaliana resistome in response to cucumber mosaic virus infection using whole genome microarray. Plant Mol Biol 55(4):501-520

Martin-Magniette ML, Aubert J, Cabannes E, Daudin JJ (2005) Evaluation of the gene-specific dye bias in cDNA microarray experiments. Bioinformatics 21(9):1995-2000

Mazarei M, Liu W, Al-Ahmad H, Arelli PR, Pantalone VR, Stewart CN (2011) Gene expression profiling of resistant and susceptible soybean lines infected with soybean cyst nematode. Theor Appl Genet 123(7):1193-1206

Mithen R, Magrath R (1992) A contribution to the life history of Plasmodiophora brassicae: secondary plasmodia development in root galls of Arabidopsis thaliana. Mycol Res 96(10):877-885

Muller P, Hilgenberg W (1986) Isomers of zeatin and zeatin riboside in clubroot tissue: evidence for trans-zeatin biosynthesis by Plasmodiophora brassicae. Physiol Plant 66(2):242-250

Neuhaus K, Grsic-Rausch S, Sauerteig S, Ludwig-Muller J (2000) Arabidopsis plants transformed with nitrilase 1 or 2 in antisense direction are delayed in clubroot development. J Plant Physiol 156(5/6):756-761

Palloix A, Ayme V, Moury B (2009) Durability of plant major resistance genes to pathogens depends on the genetic background, experimental evidence and consequences for breeding strategies. New Phytol 183(1):190-199

Pasold S, Siegel I, Seidel C, Ludwig-Muller J (2010) Flavonoid accumulation in Arabidopsis thaliana root galls caused by the obligate biotrophic pathogen Plasmodiophora brassicae. Mol Plant Pathol 11(4):545-562

Piao ZY, Ramchiary N, Lim YP (2009) Genetics of clubroot resistance in Brassica species. J Plant Growth Regul 28(3):252-264

Poland JA, Balint-Kurti PJ, Wisser RJ, Pratt RC, Nelson RJ (2009) Shades of gray: the world of quantitative disease resistance. Trends Plant Sci 14(1):21-29

Puskas LG, Zvara A, Hackler LJr, Van Hummelen P (2002) RNA amplification results in reproducible microarray data with slight ratio bias. Biotechniques 32(6):1330-1340

Puzio PS, Newe M, Grymaszewska G, Ludwig-Muller J, Grundler FMW (2000) Plasmodiophora brassicae-induced expression of pyk20, an Arabidopsis thaliana gene with glutamine-rich domain. Physiol Mol Plant Pathol 56(2):79-84

Radwan O, Liu Y, Clough SJ (2011) Transcriptional analysis of soybean root response to Fusarium virguliforme, the causal agent of sudden death syndrome. Mol Plant-Microbe Interact 24(8):958-972

Rocherieux J, Glory P, Giboulot A, Boury S, Barbeyron G, Thomas G, Manzanares-Dauleux MJ (2004) Isolate-specific and broadspectrum QTLs are involved in the control of clubroot in Brassica oleracea. Theor Appl Genet 108(8):1555-1563

Siemens J, Nagel M, Ludwig-Muller J, Sacristán MD (2002) The interaction of Plasmodiophora brassicae and Arabidopsis thaliana: parameters for disease quantification and screening of mutant lines. J Phytopathol 150(11/12):592-605

Siemens J, Keller I, Sarx J, Kunz S, Schuller A, Nagel W, Schmulling T, Parniske M, Ludwig-Muller J (2006) Transcriptome analysis of Arabidopsis clubroots indicate a key role for cytokinins in disease development. Mol Plant-Microbe Interact 19(5):480-494

Siemens J, Gonzalez MC, Wolf S, Hofmann C, Greiner S, Du YJ, Rausch T, Roitsch T, Ludwig-Muller J (2011) Extracellular invertase is involved in the regulation of clubroot disease in Arabidopsis thaliana. Mol Plant Pathol 12(3):247-262

Somé A, Manzanares MJ, Laurens F, Baron F, Thomas G, Rouxel F (1996) Variation for virulence on Brassica napus L. amongst 
Plasmodiophora brassicae collections from France and derived single-spore isolates. Plant Pathol 45(3):432-439

Swarbrick PJ, Huang K, Liu G, Slate J, Press MC, Scholes JD (2008) Global patterns of gene expression in rice cultivars undergoing a susceptible or resistant interaction with the parasitic plant Striga hermonthica. New Phytol 179(2):515-529

Tao Y, Xie ZY, Chen WQ, Glazebrook J, Chang HS, Han B, Zhu T, Zou GZ, Katagiri F (2003) Quantitative nature of Arabidopsis responses during compatible and incompatible interactions with the bacterial pathogen Pseudomonas syringae. Plant Cell 15 (2):317-330

Thatcher LF, Anderson JP, Singh KB (2005) Plant defence responses: what have we learnt from Arabidopsis? Funct Plant Biol 32(1):1-19

Thilmony R, Underwood W, He SY (2006) Genome-wide transcriptional analysis of the Arabidopsis thaliana interaction with the plant pathogen Pseudomonas syringae pv. tomato DC3000 and the human pathogen Escherichia coli O157:H7. Plant J 46(1):34-53
Thimm O, Blasing O, Gibon Y, Nagel A, Meyer S, Kruger P, Selbig J, Muller LA, Rhee SY, Stitt M (2004) MAPMAN: a user-driven tool to display genomics data sets onto diagrams of metabolic pathways and other biological processes. Plant J 37(6):914-939

van Damme M, Huibers RP, Elberse J, Van den Ackerveken G (2008) Arabidopsis DMR6 encodes a putative 2OG-Fe(II) oxygenase that is defense-associated but required for susceptibility to downy mildew. Plant J 54(5):785-793

Wagner G, Charton S, Lariagon C, Laperche A, Lugan R, Hopkins J, Frendo P, Bouchereau A, Delourme R, Gravot A, ManzanaresDauleux MJ (2012) Metabotyping: a new approach to investigate rapeseed (Brassica napus L.) genetic diversity in the metabolic response to clubroot infection. Mol Plant-Microbe Interact 25 (11):1478-1491

Wallenhammar AC (1996) Prevalence of Plasmodiophora brassicae in a spring oilseed rape growing area in central Sweden and factors influencing soil infestation levels. Plant Pathol 45(4):710-719 